\title{
ON CERTAIN PAIRS OF TRANSCENDENTAL FUNCTIONS WHOSE ROOTS SEPARATE EACH OTHER*
}

\author{
MAXIME BÔCHER
}

Let us consider eight functions $p, q, \phi_{1}, \phi_{2}, \psi_{1}, \psi_{2}, \chi_{1}, \chi_{2}$ of the real variable $x$, which throughout the interval

$$
a \leqq x \leqq b
$$

are real and continuous, and of which the last six have finite first derivatives at every point of this interval. Let $y$ be a solution, which we assume to be not identically zero, of the differential equation

$$
y^{\prime \prime}+p y^{\prime}+q y=0 \text {. }
$$

The functions whose roots we wish to consider in this paper are the following:

$$
\Phi=\phi_{2} y^{\prime}-\phi_{1} y, \quad \Psi=\psi_{2} y^{\prime}-\psi_{1} y, \quad \mathrm{X}=\chi_{2} y^{\prime}-\chi_{1} y .
$$

It should be noticed that, as a consequence of the assumptions made above, these functions are continuous and have finite first derivatives at every point of the interval $(I)$.

The roots of functions of this form were considered by STURM $\dagger$ by a method which, however, yields results distinctly less general than those we shall obtain. The relation of our methods and results to those of STURM will be indicated at the proper places.

The third section of the present paper may appear at first sight to be of slight importance, the generalizations of the results of the first two sections which are contained in it being in themselves not very far-reaching. The method used in establishing these generalizations is, however, I believe, a valuable one apart from the special application here made of it. I have indicated one application to other questions in the footnote on p. 435.

* Presented to the Society August 19, 1901. Received for publication Angust 10, 1901.

† Liouville's Journal, vol. 1 (1836), pp. 149-164. 


\section{§1. Two functions.}

We shall consider in the present section only the two functions $\Phi, \Psi$. These functions satisfy a differential equation which may be obtained as follows:

We start from the identity:

$$
\Phi\left(\psi_{2} y^{\prime}-\psi_{1} y\right)-\Psi\left(\phi_{2} y^{\prime}-\phi_{1} y\right)=0 .
$$

From this we obtain by differentiation the equation:

$$
\begin{aligned}
\left(\Phi^{\prime} \psi_{2}-\Psi^{\prime} \phi_{2}+\Phi \psi_{2}^{\prime}\right. & \left.-\Psi \phi_{2}^{\prime}-\Phi \psi_{1}+\Psi \phi_{1}-p \Phi \psi_{2}+p \Psi \phi_{2}\right) y^{\prime} \\
& -\left(\Phi^{\prime} \psi_{1}-\Psi^{\prime} \phi_{1}+\Phi \psi_{1}^{\prime}-\Psi \phi_{1}^{\prime}+q \Phi \psi_{2}-q \Psi \phi_{2}\right) y=0 .
\end{aligned}
$$

Since $y$ and $y^{\prime}$ cannot both vanish at any point of $(I)$ we can eliminate them between the last two equations. This gives :

where for the sake of abbreviation we have set

$$
\left.\Phi_{1}\right)\left(\Phi^{\prime} \Psi-\Phi \Psi^{\prime}\right)+\left\{\psi_{1}, \psi_{2}\right\} \Phi^{2}-\left\{\psi_{1}, \phi_{2}, \phi_{1}, \psi_{2}\right\} \Phi \Psi
$$

$$
+\left\{\phi_{1}, \phi_{2}\right\} \Psi^{2}=0
$$

$$
\left\{\psi_{1}, \psi_{2}\right\}=\psi_{1}^{\prime} \psi_{2}-\psi_{1} \psi_{2}^{\prime}+\psi_{1}^{2}+p \psi_{1} \psi_{2}+q \psi_{2}^{2},
$$

$$
\begin{gathered}
\left\{\psi_{1}, \phi_{2}, \phi_{1}, \psi_{2}\right\}=\psi_{1}^{\prime} \phi_{2}-\psi_{1} \phi_{2}^{\prime}+\phi_{1}^{\prime} \psi_{2}-\phi_{1} \psi_{2}^{\prime}+2 \phi_{1} \psi_{1}+p\left(\psi_{1} \phi_{2}+\phi_{1} \psi_{2}\right) \\
\left\{\phi_{1}, \phi_{2}\right\}=\phi_{1}^{\prime} \phi_{2}-\phi_{1} \phi_{2}^{\prime}+\phi_{1}^{2}+p \phi_{1} \phi_{2}+q \phi_{2}^{2} .
\end{gathered}
$$

Equation (2) is the differential equation we were seeking. It may be termed a homogeneous Riccati's equation for the pair of functions $\Phi, \Psi$.*

If the two functions $\Phi$ and $\Psi$ were to vanish at the same point we should have at this point

$$
\begin{aligned}
& \phi_{2} y^{\prime}-\phi_{1} y=0, \\
& \psi_{2} y^{\prime}-\psi_{1} y=0,
\end{aligned}
$$

and, since $y$ and $y^{\prime}$ eannot both vanish at this point, as otherwise $y$ would be identically zero, we must have at this point

$$
\phi_{1} \psi_{2}-\phi_{2} \psi_{1}=0
$$

* By a Riccati's equation is ordinarily understood an equation of the form :

$$
\omega^{\prime}=A+B \omega+C \omega^{2} \text {. }
$$

If for $\omega$ we write $\omega_{1} / \omega_{2}$ this equation takes the homogeneous form :

$$
\omega_{1}^{\prime} \omega_{2}-\omega_{1} \omega_{2}^{\prime}=A \omega_{2}^{2}+B \omega_{1} \omega_{2}+C \omega_{1}^{2},
$$

and this is the form of equation (2). Conversely if we divide (2) by $\Phi^{2}$ and $\Psi^{2}$ respectively we obtain at once the ordinary Riccati's equation for $\Psi / \Phi$ and $\Phi / \Psi$.

It should he noticed that the function $\left\{\phi_{1}, \phi_{2}\right\}$ (or $\left\{\psi_{1}, \psi_{2}\right\}$ ) when equated to zero gives the homogeneous form of the Riccati's equation which is satified by $y^{\prime} \mid y$. 
By assuming, as we shall now do, that $\phi_{1} \psi_{2}-\phi_{2} \psi_{1}$ does not vanish at any point of $(I)$ we make it impossible for $\Phi$ and $\Psi$ to vanish together.

In this section and the next we shall not use equation (2) itself, but only the following formulæ which follow from it at once:

At a point where $\Phi=0$ :

$$
\Phi^{\prime}=-\frac{\left\{\phi_{1}, \phi_{2}\right\}}{\phi_{1} \psi_{2}-\phi_{2} \psi_{1}} \Psi
$$

At a point where $\Psi=0$ :

$$
\Psi^{\prime}=\frac{\left\{\psi_{1}, \psi_{2}\right\}}{\phi_{1} \psi_{2}-\phi_{2} \psi_{1}} \Phi
$$

Since $\Phi$ and $\Psi$ cannot vanish together we see from (3) that, if $\left\{\phi_{1}, \phi_{2}\right\}$ does not vanish in $(I), \Phi$ and $\Phi^{\prime}$ cannot vanish together, and therefore $\Phi$ cannot vanish an infinite number of times in $(I)$. Whatever the functions $\phi_{1}$ and $\phi_{2}$ may be, provided merely they do not both vanish at any point of $(I)$, the functions $\psi_{1}$ and $\psi_{2}$ can be so chosen (e. g., $\psi_{1}=-\phi_{2}, \psi_{2}=\phi_{1}$ ) that $\phi_{1} \psi_{2}-\phi_{2} \psi_{1}$ does not vanish in $(I)$. Moreover if $\phi_{1}$ and $\phi_{2}$ vanished at the same point, $\left\{\phi_{1}, \phi_{2}\right\}$ would also vanish there. We have therefore the theorem which it will be noticed relates to the function $\Phi$ alone:

I. If $\left\{\phi_{1}, \phi_{2}\right\}$ does not vanish in $(I)$, then $\Phi$ cannot vanish more than a finite number of times there and $\Phi$ and $\Phi^{\prime}$ do not both vanish at any point of $(I) .{ }^{*}$

Since at two successive roots of $\Phi$ the derivative $\Phi^{\prime}$ has opposite signs, and since by (3) this can happen only when $\Psi$ has opposite signs at these two points, we have the theorem:

II. If neither of the two functions

$$
\phi_{1} \psi_{2}-\phi_{2} \psi_{1}, \quad\left\{\phi_{1}, \phi_{2}\right\}
$$

vanishes in $(I)$, then in any portion of $(I)$ in which $\Psi$ does not vanish $\Phi$ cannot vanish more than once. $\dagger$

* STURM (1. c., p. 150) proves this theorem for the special case $\phi_{2}=e^{\int p d x}$ by using the special case of formula (2) obtained by letting $\psi_{2}=0, \psi_{1}=-1$. This special case, written to be sure in a slightly modified form, is his equation (11). STURM also restricts himself to the case $\left\{\phi_{1}, \phi_{2}\right\}>0$ (see his inequality (14)) and can thus add to our theorem the statement that $\Phi^{\prime}$ and $y=\Psi$ have opposite signs when $\Phi=0$. He does not point out the connection between this formula and the Riccati's equations which he uses on p. 159.

$\dagger$ This theorem is proved by $\operatorname{STURM}(1$. o., p. 160) by a wholly different method, but only in the very special case

$$
\psi_{2}=0, \psi_{1}=-1, \phi_{2}=e^{\int p d x}, q>0, \phi_{1}^{\prime} \geqq 0 .
$$

This is the more remarkable as his formula (11) would have yielded him the result in the much more general case in which his last two conditions are replaced by the single condition $\left\{\phi_{1}\right.$, $\left.\phi_{2}\right\}>0$. This would have brought his later results ( pp. 158-164) into harmony with those which immediately precede them. 
A similar result of course holds for the roots of the function $\Psi$, and by combining these facts we get the theorem :

III. If none of the functions

$$
\phi_{1} \psi_{2}-\phi_{2} \psi_{1}, \quad\left\{\phi_{1}, \phi_{2}\right\}, \quad\left\{\psi_{1}, \psi_{2}\right\}
$$

vanish in $(I)$ then between two roots of $\Phi$ lies one and only one root of $\Psi$, and between two roots of $\Psi$ lies one and only one root of $\Phi . *$

That is, the roots of $\Phi$ and $\Psi$ separate each other.

Let us now consider two points at one of which $\Phi$ at the other $\Psi$ vanishes while neither function vanishes between them. These points are two successive roots of the product $\Phi \Psi$, and therefore the derivative $\Phi^{\prime} \Psi+\Phi \Psi^{\prime}$ has opposite signs at them. From this it follows, as a glance at (3) and (4) shows, that if none of the functions

$$
\phi_{1} \psi_{2}-\phi_{2} \psi_{1}, \quad\left\{\phi_{1}, \phi_{2}\right\}, \quad\left\{\psi_{1}, \psi_{2}\right\}
$$

vanish in $(I)$ then $\left\{\phi_{1}, \phi_{2}\right\}$ and $\left\{\psi_{1}, \psi_{2}\right\}$ must have the same sign. If, then, these two functions have opposite signs, our assumption that there exist two points, at one of which $\Phi$ at the other $\Psi$ vanishes, must be false, and, therefore, by theorem III, neither of these functions can vanish twice. Hence the theorem:

IV. If none of the functions:

$$
\phi_{1} \psi_{2}-\phi_{2} \psi_{1}, \quad\left\{\phi_{1}, \phi_{2}\right\}, \quad\left\{\psi_{1}, \psi_{2}\right\}
$$

vanish in $(I)$, and if the last two of them have opposite signs, then neither $\Phi$ nor $\Psi$ vanishes more than once in $(I)$, and if one of these functions vanishes the other does not.

A special case of this theorem deserves mention, namely that in which $\Psi=y$. In order that $\phi_{1} \psi_{2}-\phi_{2} \psi_{1}$ shall not vanish, it is necessary and sufficient that $\phi_{2}$ do not vanish in $(I)$. If then we let $\phi=\phi_{1} / \phi_{2}$ we have the theorem :

V. If $\phi$ is continuous, has a finite derivative at every point of $(I)$, and satisfies the inequality:

$$
\{\phi, 1\}=\phi^{\prime}+\phi^{2}+p \phi+q<0,
$$

then neither y nor $y^{\prime}-\phi y$ vanishes more than once in $(I)$, and if one of these functions vanishes the other does not. $\dagger$

* This theorem is also proved by StURM (1. c., pp. 161, 163) first in a case analogous to the one mentioned in the last footnote, then in the more general, but still very special, case

$$
\phi_{2}=\psi_{2}=e^{\int p d x}, q>0, \phi_{1}^{\prime} \geqq 0, \psi_{1}^{\prime} \geqq 0 .
$$

It should be noticed that the condition $\phi_{1} \psi_{2}-\phi_{2} \psi_{1} \neq 0$ now meaus that $\phi_{1}$ is either everywhere greater or every where less than $\psi_{1}$.

† The part of this theorem which says that $y$ cannot vanish more than once was proved by the writer by another method in the Bulletin of the American Mathematical Society, May, 1901, p. 335, footnote. Cf. also a less far reaching theorem of PICARD there quoted. 
Thus for instance if $q<0, y$ and $y^{\prime}$ cannot both vanish in $(I)$ nor can either of them vanish more than once.

\section{§2. Three functions.}

We will now establish the following theorem concerning the three functions $\Phi, \Psi, \mathrm{X}$.

VI. If none of the six functions :

$\phi_{1} \psi_{2}-\phi_{2} \psi_{1}, \quad \psi_{1} \chi_{2}-\psi_{2} \chi_{1}, \quad \chi_{1} \phi_{2}-\chi_{2} \phi_{1}, \quad\left\{\phi_{1}, \phi_{2}\right\}, \quad\left\{\psi_{1}, \psi_{2}\right\}, \quad\left\{\chi_{1}, \chi_{2}\right\}$ vanish in $(I)$, if the last three of them have the same sign, and if the product of all six is negative, then between any root of $\Phi$ and a larger root of $\mathrm{X}$ lies a root of $\Psi$, between any root of $\Psi$ and a larger root of $\Phi$ lies a root of $\mathrm{X}$, and between any root of $\mathrm{X}$ and a larger root of $\Psi$ lies a root of $\Phi . *$

Let us write :

$$
i=\operatorname{sgn}\left\{\phi_{1}, \phi_{2}\right\}=\operatorname{sgn}\left\{\psi_{1}, \psi_{2}\right\}=\operatorname{sgn}\left\{\chi_{1}, \chi_{2}\right\} \cdot \dagger
$$

Then the assumption that the product of the six functions is negative is expressed by the equation :

$$
i \cdot \operatorname{sgn}\left(\phi_{1} \psi_{2}-\phi_{2} \psi_{1}\right) \cdot \operatorname{sgn}\left(\psi_{1} \chi_{2}-\psi_{2} \chi_{1}\right) \cdot \operatorname{sgn}\left(\chi_{1} \phi_{2}-\chi_{2} \phi_{1}\right)=-1 .
$$

Let $x_{1}$ and $x_{2}$ be two points of $(I)$ such that

$$
\Phi\left(x_{1}\right)=0, \quad \mathrm{X}\left(x_{2}\right)=0, \quad x_{1}<x_{2} .
$$

If $\Phi$ vanished between $x_{1}$ and $x_{2}$, say at $x^{\prime}, \Psi$ would vanish, by theorem II, between $x_{1}$ and $x^{\prime}$. If $\Phi$ does not vanish between $x_{1}$ and $x_{2}$ we may proceed as follows :

Since sgn $\Phi\left(x_{1}+\epsilon\right)=\operatorname{sgn} \Phi^{\prime}\left(x_{1}\right)$ we see by formula (3) that

$$
\operatorname{sgn} \Phi\left(x_{1}+\epsilon\right)=-i \cdot \operatorname{sgn}\left(\phi_{1} \psi_{2}-\phi_{2} \psi_{1}\right) \cdot \operatorname{sgn} \Psi\left(x_{1}\right) \text {. }
$$

Furthermore if in the identical relation

$$
\left(\phi_{1} \psi_{2}-\phi_{2} \psi_{1}\right) \mathrm{X}+\left(\chi_{1} \phi_{2}-\chi_{2} \phi_{1}\right) \Psi+\left(\psi_{1} \chi_{2}-\psi_{2} \chi_{1}\right) \Phi=0
$$

we let $x=x_{2}$ so that the first term drops out, and then note the signs of the two remaining terms, we get:

$$
\operatorname{sgn} \Psi\left(x_{2}\right)=-\operatorname{sgn}\left(\psi_{1} \chi_{2}-\psi_{2} \chi_{1}\right) \cdot \operatorname{sgn}\left(\chi_{1} \phi_{2}-\chi_{2} \phi_{1}\right) \cdot \operatorname{sgn} \Phi\left(x_{2}\right) .
$$

\footnotetext{
* A special case of this theorem and the next, analogous to the ones mentioned in previous footnotes, is proved by STURM (1. c., p. 165).

t We understand with Kronecker by sgn $\Omega$ the value +1 or -1 according as $\Omega$ is positive or negative.
} 
Combining equations (5) and (7), and remembering that

we get :

$$
\operatorname{sgn} \Phi\left(x_{2}\right)=\operatorname{sgn} \Phi\left(x_{1}+\epsilon\right),
$$

$\operatorname{sgn} \Psi\left(x_{2}\right)$

$$
=i \cdot \operatorname{sgn}\left(\phi_{1} \psi_{2}-\phi_{2} \psi_{1}\right) \cdot \operatorname{sgn}\left(\psi_{1} \chi_{2}-\psi_{2} \chi_{1}\right) \cdot \operatorname{sgn}\left(\chi_{1} \phi_{2}-\chi_{2} \phi_{1}\right) \cdot \operatorname{sgn} \Psi\left(x_{1}\right) .
$$

This equation shows that $\Psi\left(x_{2}\right)$ and $\Psi\left(x_{1}\right)$ have opposite signs. Therefore $\Psi$ vanishes between $x_{1}$ and $x_{2}$-as was to be proved.

We have thus proved the first statement of our theorem and the proof of the other two statements follows at once from considerations of symmetry.

The case in which the product of the six functions considered in the last theorem is positive can be reduced to the case just considered by interchanging two of the three letters $\phi, \psi, \chi$. If, further, we remember that under the conditions of the last theorem the roots of any two of the functions $\Phi, \Psi, \mathrm{X}$ separate each other (cf. theorem III) we obtain the following theorem :

VII. If none of the six functions

$\phi_{1} \psi_{2}-\phi_{2} \psi_{1}, \quad \psi_{1} \chi_{2}-\psi_{2} \chi_{1}, \quad \chi_{1} \phi_{2}-\chi_{2} \phi_{1}, \quad\left\{\phi_{1}, \phi_{2}\right\}, \quad\left\{\psi_{1}, \psi_{2}\right\}, \quad\left\{\chi_{1}, \chi_{2}\right\}$ vanish in $(I)$, and if the last three of them have the same sign, then the roots of the functions $\Phi, \Psi, \mathrm{X}$ follow each other cyclically in the order named or in the reverse order $\mathrm{X}, \Psi, \Phi$ according as the product of the six functions above written is negative or positive.

As an application of this theorem let us consider the three functions:

$$
\Phi=y, \quad \Psi=y^{\prime}, \quad \mathrm{X}=p y^{\prime}+q y=-y^{\prime \prime} .
$$

We must here assume that the coefficients $p, q$ of the differential equation have finite first derivatives at every point of $(I)$. We have in this case:

$$
\begin{gathered}
\phi_{1} \psi_{2}-\phi_{2} \psi_{1}=-1, \quad \psi_{1} \chi_{2}-\psi_{2} \chi_{1}=q, \quad \chi_{1} \phi_{2}-\chi_{2} \phi_{1}=p, \quad\left\{\phi_{1}, \phi_{2}\right\}=1, \\
\left\{\psi_{1}, \psi_{2}\right\}=q, \quad\left\{\chi_{1}, \chi_{2}\right\}=q p^{\prime}-q^{\prime} p+q^{2}=q^{2}\left[\frac{d}{d x}\left(\frac{p}{q}\right)+1\right] .
\end{gathered}
$$

VIII. If throughout the interval $(I)$ :

$$
q>0, \frac{d}{d x}\left(\frac{p}{q}\right)>-1,
$$

and $p$ does not vanish in this interval, then the roots of $y, y^{\prime}, y^{\prime \prime}$ follow each other in the cyclic order named if $p>0$, in the reverse order if $p<0$.

As an application of this theorem let us consider the positive roots of the three Bessel's functions $J_{n}(x), J_{n+1}(x), J_{n+2}(x)$ where $n$ has any real value. Let us introduce the new independent variable $\xi=x^{2} / 4$ and let 
Then we have:*

$$
R_{m}(\xi)=\left(\frac{2}{x}\right)^{m} J_{m}(x)
$$

$$
R_{m}^{\prime}(\xi)=-R_{m+1}(\xi) .
$$

The relative position of the positive roots of $J_{n}, J_{n+1}, J_{n+2}$ is then evidently the same as that of the positive roots of $R_{n}, R_{n}^{\prime}, R_{n}^{\prime \prime}$. Now $R_{n}$ satisfies the differential equation :

$$
y^{\prime \prime}+\frac{n+1}{\xi} y^{\prime}+\frac{1}{\xi} y=0 .
$$

By applying theorem VIII to this equation we get at once the theorem :

IX. The positive roots of $J_{n}, J_{n+1}, J_{n+2}$ follow each other cyclically in the order named if $n>-1$, in the reverse order if $n<-1 . \dagger$

\section{§3. An extension of the previous results.}

We shall begin by considering the non-homogeneous linear equation of the first order:

$$
y^{\prime}+p y=r,
$$

where $p$ and $r$ are throughout the interval $(I)$ real and continuous functions of $x$.

It is well known that there exists one and only one solution of this equation which vanishes at an arbitrary point $c$ of $(I)$ and that this solution is given by the formula:

$$
y=e^{-\int_{c}^{x} p d x} \int_{c}^{x} r e^{\int_{c}^{x} p d x} d x
$$

This formula shows us that if throughout $(I) r \geqq 0$ the following relations are satisfied:

$$
\begin{array}{ll}
y \geqq 0 & (c<x \leqq b), \\
y \leqq 0 & (c>x \geqq a) .
\end{array}
$$

* Cf. Gray and Mathews : Treatise on Bessel Functions, p. 46.

† This theorem was established by the writer in the Bulletin of the American Mathematical Society, March, 1897, p. 207, although owing to an oversight the reversal of the orcler of the roots when $n<-1$ is not mentioned there. The part of this theorem which states that the roots of $J_{n}$ and $J_{n+1}$ separate each other is of earlier date, it having been stated without proof by GRAY and MATHEws (1. c.) as being probably correct, and actually proved by VAN Vheck (A merican Journal of Mathematics, vol. 19 (1897), p. 75). Among a considerable number of other proofs which, have since been given of this part of the theorem I should like to call special attention to an extremely brief and simple one by Porrer (A merican Journal of Mathematics, vol. 20 (1898), p. 199, and Bulletin of the American Mathematical Society; March, 1898, p. 274) which has recently been reproduced by H. WEBER in his edition of Riemans's Differentialgleichungen der mathematischen Physik, vol. 1 (1900), p. 166. 
If on the other hand $r \leqq 0$ these last two inequalities must be reversed. Moreover it is clear that in each of these cases $y$ will vanish at a point $x$ when and only when $r$ vanishes at all points between $c$ and $x$.

$\mathrm{X}$. If $r$ does not change sign in $(I)$ a solution of $(8)$ vanishes either at no point of $(I)$, at one and only one point, or at all the points of a connected portion of $(I)$ throughout which $r=0$, but at no other point of $(I)$. In the last two cases :

if $r \geqq 0$

$$
\begin{aligned}
& y(a) \leqq 0, \quad y(b) \geqq 0, \\
& y(a) \geqq 0, \quad y(b) \leqq 0 . *
\end{aligned}
$$$$
\text { if } r \leqq 0
$$

Let us now consider the very much more general differential equation :

$$
y^{\prime}+p(x, y) \cdot y=r(x, y),
$$

where $p$ and $r$ are continuous real functions of the two independent variables $(x, y)$ so long as $x$ remains in $(I), y$ having any value. $\dagger$ Let us suppose that $y_{1}$ is a solution of (9) which is continuous throughout $(I)$. Then $y_{1}$ is also a solution of the linear equation :

$$
y^{\prime}+p\left(x, y_{1}\right) \cdot y=r\left(x, y_{1}\right),
$$

where $p$ and $r$ are now continuous functions of $x$ only. Moreover, if $r(x, y)$ does not change sign the same will be true $a$ fortiori of $r\left(x, y_{1}\right)$. Accordingly in this case all that is said in theorem $\mathrm{X}$ about $y$ is true of every solution of (9) which is continuous throughout $(I)$.

* I should like to indicate in passing how this theorem can be used to prove the theorems con-

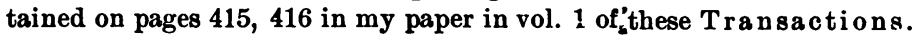

We had there two functions $\omega_{1}$ and $\omega_{2}$ continuous throughout $(I)$ and satisfying respectively the RICcati's equations :

$$
\begin{aligned}
& \omega^{\prime}=A_{1}+C_{1} \omega^{2}, \\
& \omega^{\prime}=A_{2}+C_{2} \omega^{2},
\end{aligned}
$$

where the functions $A_{1}, A_{2}, C_{1}, C_{2}$ are continuous and satisfy throughout $(I)$ the inequalities:

$$
A_{2} \geqq A_{1}, \quad C_{2} \geqq C_{1} .
$$

It is clear that the difference $\omega_{2}-\omega_{1}$ satisfies the linear equation :

$$
y^{\prime}-C_{2}\left(\omega_{2}+\omega_{1}\right) y=\left(A_{2}-A_{1}\right)+\left(C_{2}-C_{1}\right) \omega_{1}^{2} .
$$

Since $A_{2}-A_{1}+\left(C_{2}-C_{1}\right) \omega_{1}^{2} \geqq 0$, theorem X shows that if $\omega_{2}(a)>\omega_{1}(a)$ then $\omega_{2}(x)>\omega_{1}(x)$, while if $\omega_{2}(a)=\omega_{1}(a)$ then $\omega_{2}(x) \geqq \omega_{1}(x)$, and in fact $\omega_{2}(x)>\omega_{1}(x)$ provided we exclude the special cases for which throughout the neighborhood of $a, A_{2}=A_{1}$ and $\left(C_{2}-C_{1}\right) \omega_{1}^{2}=0$.

While the proof here given is perhaps a little more artificial than the one I used before, it has the advantage, besides being slightly shorter, of dispensing with the use of theorems of a not altogether elementary character about differential equations involving parameters.

† The more general case in which $p$ and $r$ are continuous only when $y$ is restricted to a finite interval might easily be considered. 
In particular the RICCATI's equation

$$
\omega^{\prime}=A+B \omega+C \omega^{2},
$$

where $A, B, C$ are continuous real functions of $x$ throughout $(I)$, is of the form (9) if we let $r=A$.

XI. If the function $A$ does not change sign in $(I)$ and does not vanish at all points of a continuous portion of $(I)$, than a real solution $\omega$ of $(10)$ which is continuous throughout $(I)$ cannot vanish at more than one point of $(I)$, and if it does vanish it passes with increasing $x$ from negative to positive if $A \geqq 0$, from positive to negative if $A \leqq 0$.

By applying this theorem to equation (2) we get the following generalization of theorem II:

XII. If $\phi_{1} \psi_{2}-\phi_{2} \psi_{1}$ does not vanish in $(I)$, and $\left\{\phi_{1}, \phi_{2}\right\}$ does not change sign and does not vanish at all points of a continuous portion of $(I)$, and if $\phi_{1}^{\prime}, \phi_{2}^{\prime}, \psi_{1}^{\prime}, \psi_{2}^{\prime}$, are continuous throughout $(I)$, then in any portion of $(I)$ in which $\Psi$ does not vanish $\Phi$ cannot vanish more than once.

From this we deduce a generalization of theorem I:

XIII. If $\phi_{1}$ and $\phi_{2}$ do not both vanish at any point of $(I)$ and if $\left\{\phi_{1}, \phi_{2}\right\}$ does not change sign in $(I)$ and does not vanish at all points of a continuous portion of $(I)$, and if $\phi_{1}^{\prime}, \phi_{2}^{\prime}$ are continuous throughout $(I)$, then $\Phi$ cannot vanish more than a finite number of times in $(I)$, and when it vanishes it changes sign.

It should he noticed that it is no longer true that $\Phi$ and $\Phi^{\prime}$ cannot vanish together. In a similar manner the other theorems of $\S \S 1,2$ can be generalized by replacing the requirement that the functions $\left\{\phi_{1}, \phi_{2}\right\},\left\{\psi_{1}, \psi_{2}\right\},\left\{\chi_{1}, \chi_{2}\right\}$ do not vanish, by the requirement that they do not change sign and do not vanish at all points of a continuous portion of $(I)$, and adding the requirement that $\phi_{1}^{\prime}$, $\phi_{2}^{\prime}, \psi_{1}^{\prime}, \psi_{2}^{\prime}, \chi_{1}^{\prime}, \chi_{2}^{\prime}$ are continuous. The proofs of some of the theorems must, however, be slightly modified. In particular, formulæ (3) and (4) must be replaced by the following:

At a point $x_{1}$ where $\Phi=0$ :

$$
\begin{aligned}
\operatorname{sgn} \frac{\Delta \Phi}{\Delta x}=\operatorname{sgn} \Phi\left(x_{1}+\epsilon\right) & =-\operatorname{sgn} \Phi\left(x_{1}-\epsilon\right) \\
& =-\operatorname{sgn}\left\{\phi_{1}, \phi_{2}\right\} \cdot \operatorname{sgn}\left(\phi_{1} \psi_{2}-\phi_{2} \psi_{1}\right) \cdot \operatorname{sgn} \Psi\left(x_{1}\right) .
\end{aligned}
$$

At a point $x_{2}$ where $\Psi=0$ :

$$
\begin{aligned}
\operatorname{sgn} \frac{\Delta \Psi}{\Delta x}=\operatorname{sgn} \Psi\left(x_{2}+\epsilon\right) & =-\operatorname{sgn} \Psi\left(x_{2}-\epsilon\right) \\
\text { GRUND IM HARZ, } & =\operatorname{sgn}\left\{\psi_{1}, \psi_{2}\right\} \cdot \operatorname{sgn}\left(\phi_{1} \psi_{2}-\phi_{2} \psi_{1}\right) \cdot \operatorname{sgn} \Phi\left(x_{2}\right) .
\end{aligned}
$$

July 20, 1901. 